\title{
Performance Rating of the Exponentiated Generalized Gompertz Makeham Distribution: An Analytical Approach
}

\author{
Ogunde Adebisi Ade ${ }^{1}$, Fatoki Olayode ${ }^{2}$, Ajayi Bamidele ${ }^{1}$ \\ ${ }^{1}$ Department of Mathematics and Statistics, The Federal Polytechnic Ado-Ekiti, Ado-Ekiti, Nigeria \\ ${ }^{2}$ Department of Statistics, Ogun State Institute of Technology, Igbesa, Nigeria
}

\section{Email address:}

debiz95@yahoo.com(Ogunde A. A.), Fatoki_olayode@yahoo.com (Fatoki O.), dele2403@gmail.com (Ajayi B.)

\section{To cite this article:}

Ogunde Adebisi Ade, Fatoki Olayode, Ajayi Bamidele. Performance Rating of the Exponentiated Generalized Gompertz Makeham Distribution: An Analytical Approach. American Journal of Theoretical and Applied Statistics. Vol. 6, No. 5, 2017, pp. $228-235$. doi: $10.11648 /$ j.ajtas.20170605.12

Received: April 22, 2017; Accepted: May 2, 2017; Published: September 4, 2017

\begin{abstract}
We developed a five parameter distribution known as the Generalized Exponentiated Gompertz Makeham distribution which is quite flexible and can have a decreasing, increasing and bathtub-shaped failure rate function depending on its parameters making it more effective in modeling survival data and reliability problems. Some comprehensive properties of the new distribution, such as closed-form expressions for the density function, cumulative distribution function, hazard rate function, moment generating function and order Statistics were provided as well as maximum likelihood estimation of the Generalized Exponentiated Gompertz Makeham distribution parameters and at the end, in order to show the distribution flexibility, an application using a real data set was presented.
\end{abstract}

Keywords: Generalized Exponentiated Gompertz Makeham Distribution, Maximum Likelihood Estimation, Bathtub-Shape Failure Rate, Distribution Flexibility

\section{Introduction}

Generalized Exponentiated Class of distribution

Cordeiro G. M. et al. Proposed a new method of adding two shape parameters to a continuous distribution that extends an idea which was first introduced by Lehmann and studied by Nadarajah and Kotz. The idea produces a new class of exponentiated generalized distributions that can be interpreted as a double construction of Lehmann alternatives. Given a continuous cumulative density function, G. M Cordeiro et al define the exponentiated generalized class of distribution by

$$
F(x)=\left[1-[1-G(x)]^{a}\right]^{b}
$$

And the probability density function given by

$$
f(x)=a b[G(x)]^{a-1}\left[1-[G(x)]^{a}\right]^{b-1} g(x)
$$

Where are two additional shape parameters in equations can control the both the tail weight and possibly adding entropies to the center of the exponentiated generalized density function.

\section{Gompertz Makeham Distribution}

The Gompertz distribution was first introduced by Benjamin Gompertz a British actuary. The distribution has been used frequently to describe human mortality, growth model and actuarial tables.

A different version of Gompertz distribution which is called Gompertz Makeham (GM) distribution was introduced by another British actuary, Makeham. He introduced a constant (Makeham terms) that describe the age independent mortality and has received considerable attention in the literature. The GM family has been studied by Baily et al. and an expression using the Lambert $\mathrm{W}$ function for the quantile function was given by Jodra, P. Suppose now is a GM random variable with the cumulative density function given by

$$
G(x)=1-e^{-\lambda x-\frac{\alpha}{\beta}\left(e^{\beta x}-1\right)}
$$

And the probability density function given by 


$$
g(x)=\left(\lambda+\alpha e^{\beta x}\right) e^{-\lambda x-\frac{\alpha}{\beta}\left(e^{\beta x}-1\right)} \quad \alpha, \beta, \lambda>0
$$

According to Finch, the Gompertz Makeham distribution produces a better fit between the age windows 30 to 85 years. An extension of the distribution will induce flexibility and enable it to cope with early failure or infant mortality.

\section{The Proposed Generalized Exponentiated Gompertz Makeham Distributions}

Putting (3) in (1), the cumulative density function of generalized exponentiated Gompertz Makeham (EGGM)

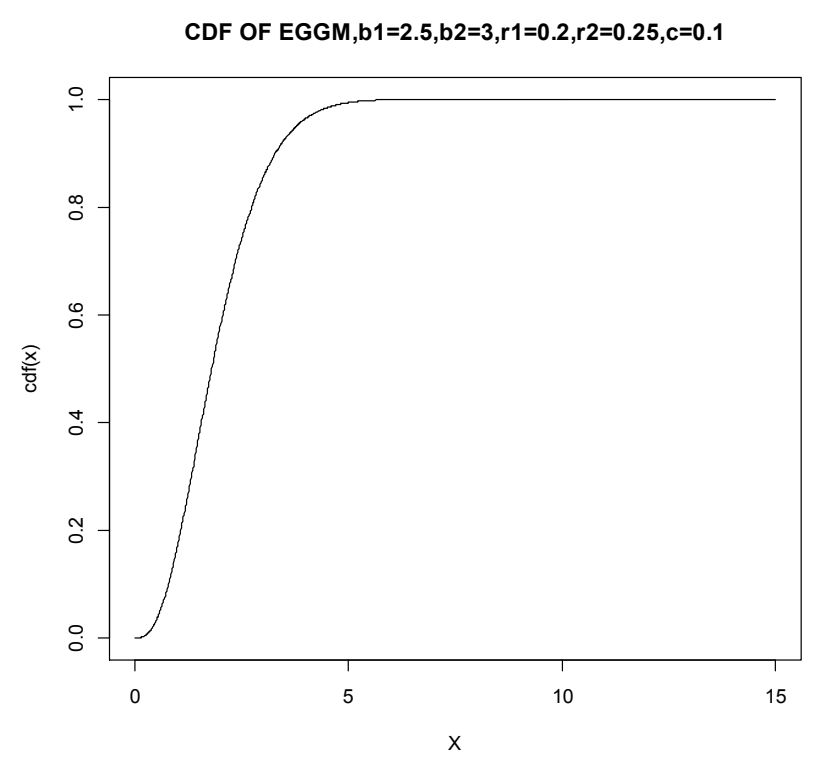

distribution can be obtained as follows

$$
\left.F(x)=\left[1-e^{a\left[-\lambda x-\frac{\alpha}{\beta}\left(e^{\beta x}-1\right)\right.}\right]\right]^{b}
$$

The graph below depicts the behaviour of the Cumulative density function of the EGGM distribution.

Also putting (4) in (2), we obtain an expression for the probability density function of the Generalized Exponentiated Gompertz Makeham (EGGM) distribution as follows

$$
f(x)=a b\left(\lambda+\alpha e^{\beta x}\right) e^{a\left[-\lambda x-\frac{\alpha}{\beta}\left(e^{\beta x}-1\right)\right]}\left[1-e^{a\left[-\lambda x-\frac{\alpha}{\beta}\left(e^{\beta x}-1\right)\right]}\right]^{b-1}
$$

The graph below depicts the behaviour of EGGM at different values of the shape parameters.

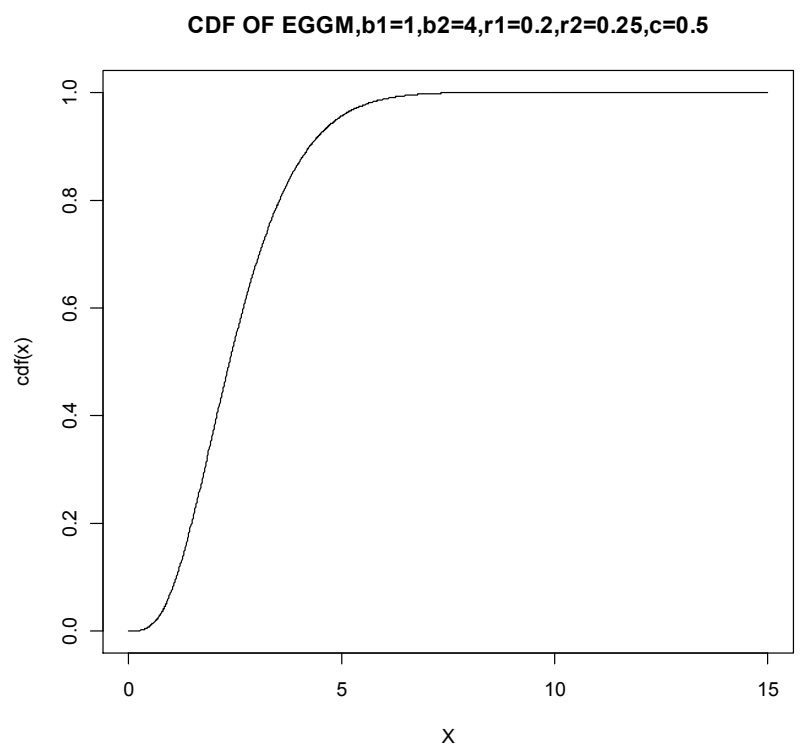

Figure 1. The cdf of EGGM for various parameters.
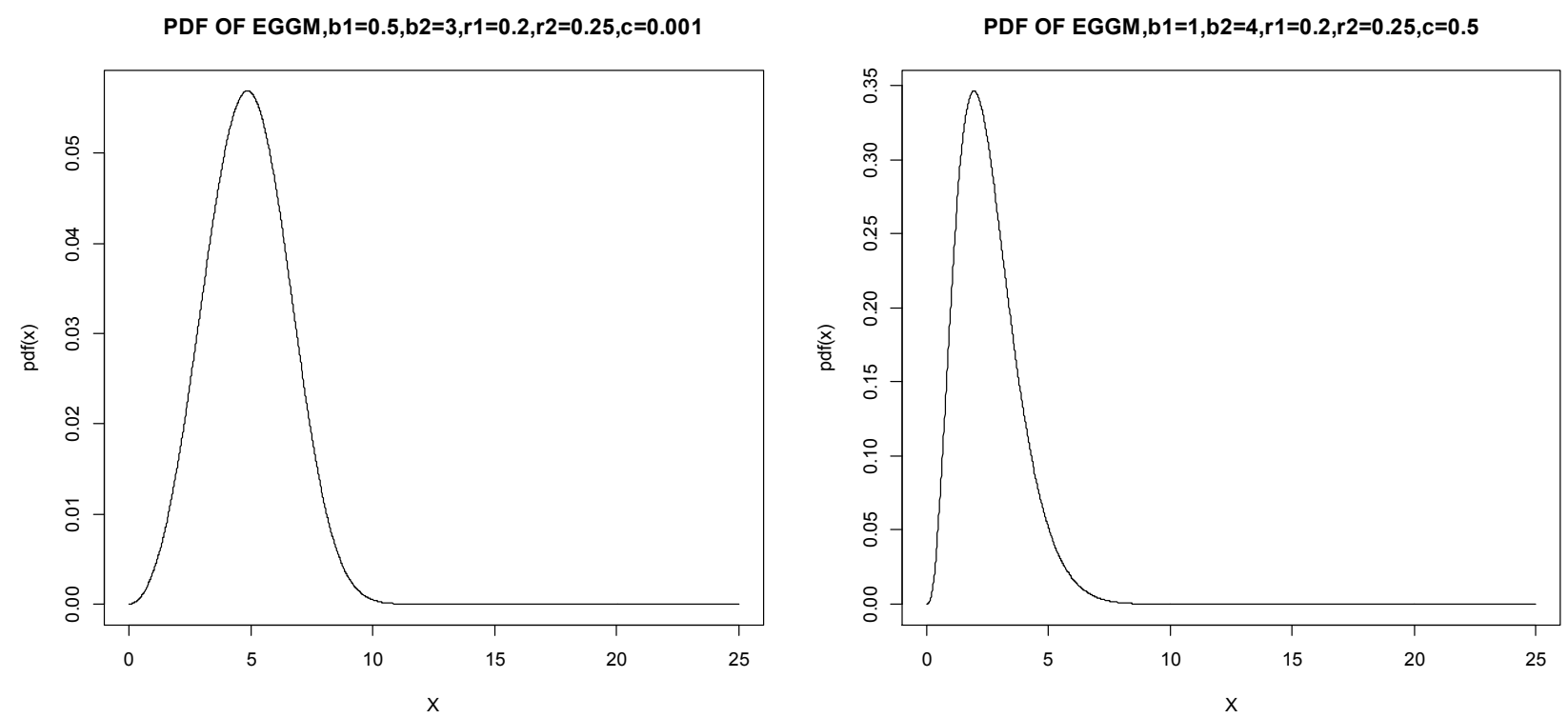

Figure 2. The pdf of EGGM for various parameters. 
The graph drawn above indicates that the pdf of EGGM is positively skewed

\subsection{Expansion for the Density Function}

For any real non integer b, we consider the binomial series,

$$
(1-z)^{b}=\quad \sum_{k=0}^{\infty}(-1)^{k}\left(\begin{array}{l}
b \\
k
\end{array}\right) z^{k}
$$

Which is valid for $|z|<1$

Applying equation (7) in (5), we have

$$
F(x)=\sum_{j=0}^{\infty}(-1)^{j}\left(\begin{array}{l}
b \\
j
\end{array}\right) e^{a\left[-\lambda x-\frac{\alpha}{\beta}\left(e^{\beta x}-1\right)\right] j}
$$

Also for the probability density function we have

$f(x)=a b\left(\lambda+\alpha e^{\beta x}\right) e^{a\left[-\lambda x-\frac{\alpha}{\beta}\left(e^{\beta x}-1\right)\right]} \sum_{j=0}^{\infty}(-1)^{j}\left(\begin{array}{c}b-1 \\ j\end{array}\right) e^{a\left[-\lambda x-\frac{\alpha}{\beta}\left(e^{\beta x}-1\right)\right] j}$

Finally we have

$$
f(x)=\operatorname{abg}(x) \sum_{j=0}^{\infty}(-1)^{j}\left(\begin{array}{c}
b-1 \\
j
\end{array}\right) e^{\left[-\lambda x-\frac{\alpha}{\beta}\left(e^{\beta x}-1\right)\right](a+a j-1)}
$$

\subsection{Verification of Exponentiated Generalized Distribution to Be a Proper Pdf}

Here, we want to show that the integral of the EGGM distribution equal to 1 ; that is

$$
\begin{gathered}
\int_{-\infty}^{\infty} f(x)=1 \\
\int_{-\infty}^{\infty} f(x)=\int_{-\infty}^{\infty} a b\left(\lambda+\alpha e^{\beta x}\right) e^{a\left[-\lambda x-\frac{\alpha}{\beta}\left(e^{\beta x}-1\right)\right]}[1- \\
\left.e^{a\left[-\lambda x-\frac{\alpha}{\beta}\left(e^{\beta x}-1\right)\right]}\right]^{b-1} d x
\end{gathered}
$$

Let $M=e^{-\lambda x-\frac{\alpha}{\beta}\left(e^{\beta x}-1\right)}$, then

$d M=-\left(\lambda+\alpha e^{\beta x}\right) e^{-\lambda x-\frac{\alpha}{\beta}\left(e^{\beta x}-1\right)}$,

this implies that $d x=\frac{-d M}{\left(\lambda+\alpha e^{\beta x}\right) M}$ substitute this in equation (12), we have

$$
\int_{-\infty}^{\infty} f(x)=\int_{-\infty}^{\infty} a b\left(\lambda+\alpha e^{\beta x}\right) M^{a}\left(1-M^{a}\right)^{b-1} \frac{d M}{\left(\lambda+\alpha e^{\beta x}\right) M}
$$

On simplification this gives

$$
\int_{-\infty}^{\infty} f(x)=a b \int_{-\infty}^{\infty} M^{a-1}\left(1-M^{a}\right)^{b-1} d M
$$

Further if we let $P=M^{a}, d P=a M^{a-1} d M, d M=\frac{d P}{a M^{a-1}}$, substitute this in equation (13), we have

$$
\int_{-\infty}^{\infty} f(x)=a b \int_{-\infty}^{\infty}\left(P^{\frac{1}{a}}\right)^{a-1}(1-P)^{b-1} \frac{d P}{a\left(P^{\frac{1}{a}}\right)^{a-1}},
$$

Finally we have

$$
\int_{-\infty}^{\infty} f(x)=\int_{-\infty}^{\infty} b(1-P)^{b-1} d P=1
$$

This verified that the pdf of EGGM distribution function is a proper pdf.

\subsection{Investigation of the Asymptotic Properties of EEGM Distribution}

We seek to investigate the behaviour of the model in Equation (6) as $x \rightarrow 0$

We have

$$
\begin{gathered}
\lim _{x \rightarrow 0} b\left(\lambda+\alpha e^{\beta x}\right) e^{\left[-\lambda x-\frac{\alpha}{\beta}\left(e^{\beta x}-1\right)\right]}\left[1-e^{\left[-\lambda x-\frac{\alpha}{\beta}\left(e^{\beta x}-1\right)\right]}\right]^{b-1} \\
=b(\lambda+\alpha)
\end{gathered}
$$

Also as $x \rightarrow 0$ and $b=1$, we have

$$
\begin{gathered}
\lim _{x \rightarrow 0} a\left(\lambda+\alpha e^{\beta x}\right) e^{a\left[-\lambda x-\frac{\alpha}{\beta}\left(e^{\beta x}-1\right)\right]} \\
=a(\boldsymbol{\lambda}+\boldsymbol{\alpha})
\end{gathered}
$$

It has been shown that as $x \rightarrow 0, a=1, b=1$ the EGGM distribution depends mainly on the shape parameters namely, $a, b, \alpha, \beta$.

\section{Well-known Distributions That Are Special Cases of the EGGM}

(i) If then we get the EGM distribution

(ii) If, then we get, GM distribution

(iii) If $a=1, b=1, \beta \rightarrow 0$, then we get the E distribution.

(iv) If $b=1, \beta \rightarrow 0$, then we get the GE distribution which is introduced by Gupta \& Kundu (1999)

(v) If $b=1$, then we get the GG distribution which is introduced by El-Gohary \& Al-Otaibi (2013).

(vi) If $a=1, b=1$, then we get the $\mathrm{G}$ distribution. (ii)

\section{Hazard Function}

The hazard function is define as

$$
h(x)=\frac{f(x)}{1-F(x)}
$$

Putting equation (5) and (6) in (14) we obtain the hazard function of the EGGM distribution as

$$
h(x)=\frac{\left.a b\left(\lambda+\alpha e^{\beta x}\right) e^{a\left[-\lambda x-\frac{\alpha}{\beta}\left(e^{\beta x}-1\right)\right.}\right]\left[1-e^{a\left[-\lambda x-\frac{\alpha}{\beta}\left(e^{\beta x}-1\right)\right]}\right]^{b-1}}{\left.1-\left[1-e^{a\left[-\lambda x-\frac{\alpha}{\beta}\left(e^{\beta x}-1\right)\right.}\right]\right]^{b}}
$$

Equation (15) above can also be called the Exponentiated Generalized Gompertz Makeham model. 
Putting $a=b=1$ in equation (15); it will reduce to

$$
h(x)=\frac{\left(\lambda+\alpha e^{\beta x}\right) e^{\left[-\lambda x-\frac{\alpha}{\beta}\left(e^{\beta x}-1\right)\right]}}{e^{a\left[-\lambda x-\frac{\alpha}{\beta}\left(e^{\beta x}-1\right)\right]}}
$$

Finally,

$$
h(x)=\left(\lambda+\alpha e^{\beta x}\right)
$$

Equation (16) represents the Gompertz Makeham model. The reliability function can be obtained as

$$
\mathcal{R}(x)=1-F(x)
$$

Putting equation (5) in (17) we obtain the reliability

$$
\begin{gathered}
M_{x}(t)=E\left(e^{t x}\right)=\int_{-\infty}^{\infty} e^{t x} f(x) d x, \quad \text { where }|t|<1 \\
M_{x}(t)=\int_{0}^{\infty} e^{t x} a b\left(\lambda+\alpha e^{\beta x}\right) \sum_{j=0}^{\infty}(-1)^{j}\left(\begin{array}{c}
b-1 \\
j
\end{array}\right) e^{a\left[-\lambda x-\frac{\alpha}{\beta}\left(e^{\beta x}-1\right)\right](j+1)} d x
\end{gathered}
$$

This can be simplified as

$$
M_{x}(t)=a b \sum_{j=0}^{\infty}(-1)^{j}\left(\begin{array}{c}
b-1 \\
j
\end{array}\right) \int_{0}^{\infty}\left(\lambda+\alpha e^{\beta x}\right) e^{t x+a\left[-\lambda x-\frac{\alpha}{\beta}\left(e^{\beta x}-1\right)\right](j+1)} d x
$$

Where

$$
\int_{-\infty}^{\infty}\left(\lambda+\alpha e^{\beta x}\right) e^{t x+a\left[-\lambda x-\frac{\alpha}{\beta}\left(e^{\beta x}-1\right)\right](j+1)} d x=\int_{0}^{\infty} \lambda e^{t x+a\left[-\lambda x-\frac{\alpha}{\beta}\left(e^{\beta x}-1\right)\right](j+1)} d x+\int_{0}^{\infty} \alpha e^{t x+\beta x+a\left[-\lambda x-\frac{\alpha}{\beta}\left(e^{\beta x}-1\right)\right](j+1)} d x
$$

We let,

$$
\begin{gathered}
I_{1}=\int_{0}^{\infty} \lambda e^{t x+a\left[-\lambda x-\frac{\alpha}{\beta}\left(e^{\beta x}-1\right)\right](j+1)} d x \\
\text { and } \quad I_{2}=\int_{0}^{\infty} \alpha e^{t x+\beta x+a\left[-\lambda x-\frac{\alpha}{\beta}\left(e^{\beta x}-1\right)\right](j+1)} d x
\end{gathered}
$$

Solving for $I_{1}$ we have

$$
\begin{aligned}
& I_{1}=\lambda \int_{0}^{\infty} e^{t x+a\left[-\lambda x-\frac{\alpha}{\beta}\left(e^{\beta x}-1\right)\right](j+1)} d x \\
& I_{1}=\lambda\left[\frac{e^{t x+a\left[-\lambda x-\frac{\alpha}{\beta}\left(e^{\beta x}-1\right)\right](j+1)}}{t-a\left(\lambda+\alpha e^{\beta x}\right)(i+j)}\right]_{0}^{\infty}
\end{aligned}
$$

Then we have,

$$
I_{1}=-\left[\frac{\lambda}{t-a(\lambda+\alpha)(i+j)}\right]
$$

Also for $I_{2}$, we have

$$
I_{2}=\alpha \int_{0}^{\infty} e^{t x+\beta x+a\left[-\lambda x-\frac{\alpha}{\beta}\left(e^{\beta x}-1\right)\right](j+1)} d x
$$




$$
I_{1}=\alpha\left[\frac{e^{t x+\beta x+a\left[-\lambda x-\frac{\alpha}{\beta}\left(e^{\beta x}-1\right)\right](j+1)}}{t+\beta-a\left(\lambda+\alpha e^{\beta x}\right)(i+j)}\right]_{0}^{\infty}
$$

Therefore,

$$
I_{2}=-\left[\frac{\alpha}{t+\beta-a(\lambda+\alpha)(i+j)}\right]
$$

Finally the moment generating function of EGGM distribution is given as

$$
M_{x}(t)=-a b \sum_{j=0}^{\infty}(-1)^{j}\left(\begin{array}{c}
b-1 \\
j
\end{array}\right)\left[\frac{\lambda}{t-a(\lambda+\alpha)(i+j)}+\frac{\alpha}{t+\beta-a(\lambda+\alpha)(i+j)}\right]
$$

Order Statistics

The density $f_{i: n}(x)$ of the ith order statistics for $i=1,2, \ldots, n$ from the independent identically distributed random variable $Y_{1, . .} Y_{n}$ is given by

$$
f_{i: n}(x)=\frac{f(x)}{B(i, n-i-1)} F(x)^{i-1}[1-F(x)]^{n-i}
$$

Substituting equation (5) and (6) in equation (23), we obtain the ith order statistics of EGGM which is given as

$$
\left.\left.f_{i: n}(x)=\frac{a b\left(\lambda+\alpha e^{\beta x}\right)}{B(i, n-i-1)} e^{a\left[-\lambda x-\frac{\alpha}{\beta}\left(e^{\beta x}-1\right)\right]}\left[1-e^{a\left[-\lambda x-\frac{\alpha}{\beta}\left(e^{\beta x}-1\right)\right.}\right]\right]^{b-1}\left[\left\{1-e^{a\left[-\lambda x-\frac{\alpha}{\beta}\left(e^{\beta x}-1\right)\right.}\right]\right\}^{b}\right]^{i-1}
$$

For $\mathrm{b}$ real non-integer by applying equation (7) and let $\xi=-\lambda x-\frac{\alpha}{\beta}\left(e^{\beta x}-1\right)$, we have

$$
f_{i: n}(x)=\frac{a b\left(\lambda+\alpha e^{\beta x}\right)}{B(i, n-i-1)} e^{a \xi} \sum_{k=0}^{\infty}(-1)^{k}\left(\begin{array}{c}
b-1 \\
k
\end{array}\right) e^{a k \xi} \sum_{l=0}^{\infty}(-1)^{l}\left(\begin{array}{c}
b(i-1) \\
l
\end{array}\right) e^{a l \xi} \sum_{m=0}^{\infty}(-1)^{m}\left(\begin{array}{c}
n-i \\
m
\end{array}\right)\left[1-e^{a \xi}\right]^{b m}
$$

Further simplification we have,

$$
f_{i: n}(x)=\frac{a b\left(\lambda+\alpha e^{\beta x}\right)}{B(i, n-i-1)} e^{a \xi} \sum_{k=0}^{\infty}(-1)^{k}\left(\begin{array}{c}
b-1 \\
k
\end{array}\right) e^{a k \xi} \sum_{l=0}^{\infty}(-1)^{l}\left(\begin{array}{c}
b(i-1) \\
l
\end{array}\right) e^{a l \xi} \sum_{m=0}^{\infty}(-1)^{m}\left(\begin{array}{c}
n-i \\
m
\end{array}\right) \sum_{p=0}^{\infty}(-1)^{p}\left(\begin{array}{c}
b m \\
p
\end{array}\right) e^{a \xi p}
$$

Finally, we have

$$
f_{i: n}(x)=\frac{a b\left(\lambda+\alpha e^{\beta x}\right)}{B(i, n-i-1)} e^{a \xi} \sum_{k=0}^{\infty}(-1)^{k+l+m+p}\left(\begin{array}{c}
b-1 \\
k
\end{array}\right)\left(\begin{array}{c}
b(i-1) \\
l
\end{array}\right)\left(\begin{array}{c}
n-i \\
m
\end{array}\right)\left(\begin{array}{c}
b m \\
p
\end{array}\right) e^{a \xi(1+k+l+p)}
$$

\section{Estimation of Statistical Inference}

Let $x_{1}, x_{2}, \ldots, x_{n}$ be random variable distributed according to (8) the likelihood function of a vector of parameters given as $\Omega(a, b, \alpha, \beta, \lambda)$.

$$
\begin{gathered}
l(\Omega)=n \log (a)+n \log (b)+\sum_{i=1}^{n} \log \left[\left(\lambda+\alpha e^{\beta x_{i}}\right) e^{-\lambda x_{i}-\frac{\alpha}{\beta}\left(e^{\beta x_{i}-1}\right)}\right]+(a-1) \sum_{i=1}^{n} \log \left[e^{-\lambda x_{i}-\frac{\alpha}{\beta}\left(e^{\beta x_{i}}-1\right)}\right]+(b- \\
\text { 1) } \sum_{i=1}^{n} \log \left[1-\left[e^{-\lambda x_{i}-\frac{\alpha}{\beta}\left(e^{\beta x_{i-1}}\right)}\right]^{a}\right]
\end{gathered}
$$

Then the score vector $\nabla l=\frac{\delta l}{\delta a}, \frac{\delta l}{\delta b}, \frac{\delta l}{\delta \alpha}, \frac{\delta l}{\delta \lambda}, \frac{\delta l}{\delta \beta}$ has components,

let $\phi=-\lambda x_{i}-\frac{\alpha}{\beta}\left(e^{\beta x_{i}}-1\right)$

$$
\begin{gathered}
\frac{\delta l}{\delta a}=\frac{n}{a}+\sum_{i=1}^{n} \log (\phi)\left[\frac{(b-1) e^{\phi a}}{1-e^{\phi a}}\right] \\
\frac{\delta l}{\delta b}=\frac{n}{b}+\sum_{i=1}^{n} \log \left[1-e^{\phi a}\right] \\
\frac{\delta l}{\delta \lambda}=\sum_{i=1}^{n} \frac{e^{\phi}-x_{i} e^{\phi}\left(\alpha e^{\beta x_{i}}+\lambda\right)}{\left(\alpha e^{\beta x_{i}}+\lambda\right) e^{\phi}}+(a-1) x+\frac{a(b-1)-\left[1-e^{\phi}\right]^{a-1} x_{i} e^{\phi}}{1-e^{\phi a}}
\end{gathered}
$$




$$
\begin{aligned}
& \frac{\delta l}{\delta \alpha}=\sum_{i=n}^{n} \frac{e^{\beta x_{i}+\phi}-\lambda\left\{\frac{1}{\beta}\left(e^{\beta x_{i}}-1\right)\right\} e^{\phi}-\alpha\left\{\frac{1}{\beta}\left(e^{\beta x_{i-1}}\right)\right\} e^{\beta x_{i}+\phi}}{\left(\alpha e^{\left.\beta x_{i}+\lambda\right) e^{\phi}}\right.}-\frac{(a-1)\left\{\frac{1}{\beta}\left(e^{\left.\beta x_{i}-1\right)}\right) e^{\phi}\right.}{e^{\phi}}+\frac{a(b-1)-\left[1-e^{\phi}\right]^{a-1}\left\{\frac{1}{\beta}\left(e^{\beta x_{i}-1}\right)\right\} e^{\phi}}{1-e^{\phi a}}
\end{aligned}
$$

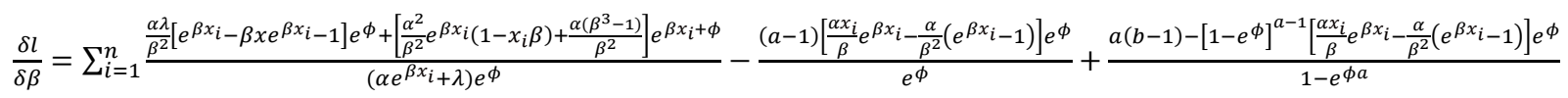

\section{Application}

To illustrate the new results presented in this paper, we fit the EGGM distribution to an uncensored data set from Nichols and Padgett, (2006) considering 100 observations on breaking stress of carbon fibres (in Gba). The data are as follows: 3.7 , $2.74,2.73,2.5,3.6,3.11,3.27,2.87,1.47,3.11,4.42,2.41$, $3.19,3.22,1.69,3.28,3.09,1.87,3.15,4.9,3.75,2.43,2.95$, $2.97,3.39,2.96,2.53,2.67,2.93,3.22,3.39,2.81,4.2,3.33$, $2.55,3.31,3.31,2.85,2.56,3.56,3.15,2.35,2.55,2.59,2.38$, $2.81,2.77,2.17,2.83,1.92,1.41,3.68,2.97,1.36,0.98,2.76$, $4.91,3.68,1.84,1.59,3.19,1.57,0.81,5.56,1.73,1.59,2,1.22$,
$1.12,1.71,2.17,1.17,5.08,2.48,1.18,3.51,2.17,1.69,1.25$, $4.38,1.84,0.39,3.68,2.48,0.85,1.61,2.79,4.7,2.03,1.8$, $1.57,1.08,2.03,1.61,2.12,1.89,2.88,2.82,2.05,3.65$. These data were previously studied by Souza et al. for beta Frechet (BF), exponentiated Frechet (EF) and Frechet distributions. In the following, we shall compare the proposed KGM and its sub-model (GM) with several other three- and four-parameter lifetime distributions, namely: the Zografos-Balakrishnan loglogistic (ZBLL), Kumaraswamy Pareto (KP) and recently the Kumaraswamy Gompertz Makeham (KGM) distribution with corresponding densities:

Where

$$
\begin{aligned}
f_{K G M}(x, a, b, \beta, \alpha, \lambda)= & a b\left(\alpha e^{\beta x}+\lambda\right)\left(e^{-\lambda x-\frac{\alpha}{\beta}\left(e^{\beta x}-1\right)}\right)\left(1-e^{-\lambda x-\frac{\alpha}{\beta}\left(e^{\beta x}-1\right)}\right)^{a-1} \\
& \left(1-\left(1-e^{-\lambda x-\frac{\alpha}{\beta}\left(e^{\beta x}-1\right)}\right)^{a}\right)^{b-1} \\
f_{Z B L L}(x, a, \beta, \theta)= & \frac{\beta}{\theta^{\beta} \tau(a)} x^{\beta-1}\left(1+\left(\frac{x}{\theta}\right)^{\beta}\right)^{-2}\left[\ln \left(1+\left(\frac{x}{\theta}\right)^{\beta}\right)\right]^{a-1} x>0 \\
f_{B F}(x, a, b, \theta, \beta)= & \frac{\beta \theta^{\beta}}{B(a, b)} x^{-(\beta+1)} e^{-a\left(\frac{\theta}{x}\right) \beta}\left(1-e^{\left.-\left(\frac{\theta}{x}\right) \beta\right)^{b-1}} x>0\right. \\
f_{K P}(x, a, b, \theta, \beta) & =a b \beta \theta^{\beta} x^{-(\beta+1)}\left[1-\left(\frac{\theta}{x}\right)^{\beta}\right]^{a-1}\left[1-\left(1-\left(\frac{\theta}{x}\right)^{\beta}\right)^{a}\right]^{b-1}
\end{aligned}
$$

Where $a, b, \beta, \theta, \alpha, \lambda>0$

Table1 gives the descriptive statistics of the data and Table 2 gives the likelihood ratio estimates of the parameters and table 3 gives the values of AIC, BIC, CAIC and HQIC for EGGM, KGM, GM, BF, KP, ZBLL, BF and EF distributions, the corresponding errors(given in parenthesis) and the statistics $l(\hat{\theta})$ (where $l(\hat{\theta})$ denotes the log-likelihood function evaluated at the maximum likelihood estimates), Akaike information criterion (AIC), the Bayesian information criterion (BIC), Consistent Akaike information criterion (CAIC) and Hannan-Quinn information criterion (HQIC). We also construct the Total Time on Test (TTT) plot

\begin{tabular}{|c|c|c|c|c|c|}
\hline ModeI & Estimates & & & & \\
\hline$E G G M$ & 4.1581 & 4.1757 & 0.5705 & $10^{-11}$ & 0.55113 \\
\hline$(a, b, \theta, \beta)$ & $(1.6264)$ & (1.6094) & $(0.4949)$ & $(0.0037)$ & $(-)$ \\
\hline$K G M$ & 3.2590 & 6.7422 & $10 e^{-11}$ & 0.22148 & 0.130941 \\
\hline$(a, b, \lambda, \alpha, \beta)$ & $(1.8545)$ & $(1.18545)$ & $(17.4572)$ & $(0.74510)$ & $(0.71868)$ \\
\hline$K P$ & 4.69523 & 236.2335 & 0.39 & 0.19204 & - \\
\hline$(a, b, \theta, \beta)$ & $(0.502)$ & (149.552) & - & $(0.045)$ & \\
\hline$Z B L L$ & 1.5501 & 1.90903 & 3.61259 & - & - \\
\hline$B F$ & 0.42934 & 138.0664 & 34.38484 & 0.72474 & - \\
\hline$(a, b, \theta, \beta)$ & $(0.236)$ & (113.552) & (21.52) & $(0.19)$ & \\
\hline$G M$ & $10^{-11}$ & 0.076941 & 0.790997 & - & - \\
\hline$(\lambda, \alpha, \beta)$ & $(0.0829)$ & $(0.03399)$ & $(0.10837)$ & & \\
\hline
\end{tabular}
for the data as well as its empirical density and cumulative density function.

Table 1. Descriptive Statistics on Breaking stress of Carbon fibres.

\begin{tabular}{lllllllc}
\hline Min & $Q_{1}$ & Med. & mean & $Q_{3}$ & Max & kurtosis & Skewness \\
\hline 0.390 & 1.840 & 2.700 & 2.6214 & 3.220 & 5.560 & 0.10494 & 0.36815 \\
\hline
\end{tabular}

Table 2. Likelihood Estimates of Parameters. 
Table 3. Criteria for Comparison.

\begin{tabular}{|c|c|c|c|c|c|}
\hline ModeI & $\boldsymbol{l}(\widehat{\boldsymbol{\theta}})$ & $A I C$ & $B I C$ & HQIC & CAIC \\
\hline $\begin{array}{l}E G G M \\
(a, b, \theta, \beta)\end{array}$ & -29.548 & 69.096 & 82.122 & 74.368 & 69.734 \\
\hline $\begin{array}{l}K G M \\
(a, b, \lambda, \alpha, \beta)\end{array}$ & -141.332 & 292.664 & 305.690 & 297.936 & 293.599 \\
\hline $\begin{array}{l}K P \\
(a, b, \theta, \beta)\end{array}$ & -166.751 & 339.502 & 347.318 & 338.084 & 339.923 \\
\hline $\begin{array}{l}Z B L L \\
(a, \theta, \beta)\end{array}$ & -162.913 & 331.826 & 339.642 & 330.408 & 332.076 \\
\hline $\begin{array}{l}B F \\
(a, b, \theta, \beta)\end{array}$ & -142.866 & 293.733 & 304.154 & 291.842 & 294.154 \\
\hline $\begin{array}{l}G M \\
(\lambda, \alpha, \beta)\end{array}$ & -149.125 & 304.25 & 312.066 & 307.413 & 304.50 \\
\hline
\end{tabular}



Figure 3. The graph of Total Time on Test Plot for the breaking stress of carbon data.

\section{Empirical density}

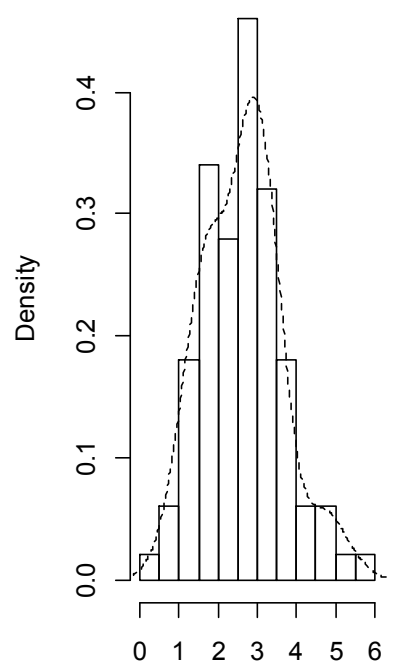

Data

\section{Cumulative distribution}

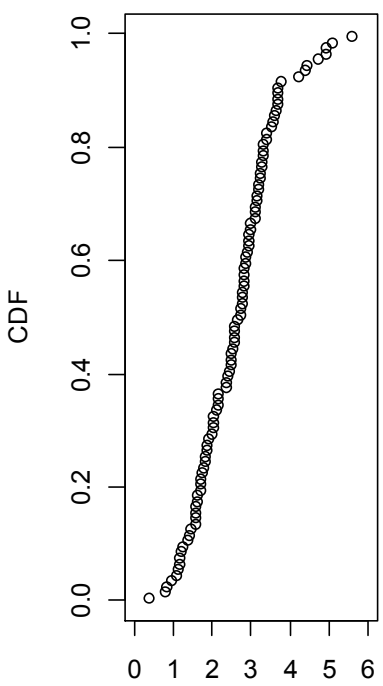

Data

Figure 4. The graph of the Emprical density and the cummulative density of the carbon data. 


\section{Conclusion}

Since the EGGM distribution has the lowest, AIC, BIC, CAIC and HQIC values among all other models and its submodel so it could be chosen as the best model.

\section{References}

[1] Bailey RC (1978) Limiting form of the Makeham model and their use for survival analysis of transplant studies. Biometries 34: 725-726.

[2] Bourguignon, M., R. B. Silva, L. M. Zea and G. M. Cordeiro, 2013. The Kumaraswamy Pareto distribution. J. of Stat. Theory and Applications, 12: 129-144.

[3] Cordeiro, G. M., E. M. M. Ortega and Daniel C. C da Cunha. The Exponentiated Generalized Class of Distributions. Journal of Data Science 11(2013), 1-27.

[4] Cordeiro, G. M. and M. de Castro, 2010. A new family of generalized distributions. Journal of Statistical Computation and Simulation, 81: 883-898.

[5] Cordeiro, G. M., et al. The Exponentiated Generalized Class of Distributions. Journal of Data Science 11(2013), 1-27.

[6] Chukwu A. U. \& Ogunde A. A. (2015), 'On the Beta Makeham Distribution and its Applications', American Journal of Mathematics and Statistics 2015, 5(3): 137-143.

[7] El-Gohary, A. \& Al-Otaibi, A. N. (2013), 'The generalized Gompertz distribution', Applied Mathematical Modeling 37(12), 13-24.

[8] Finch CE. Chicago: University of Chicago Press; 1990. Longevity. Senescence, and the Genome.

[9] Gupta, R. C., Gupta, P. L. and Gupta, R. D. (1998). Modeling failure time data by Lehmann alternatives. Communications in Statistics - Theory and Methods 27, 887-904.
[10] Gupta, R. D. and Kundu, D. (1999). Generalized exponential distributions. Australian and New Zealand Journal of Statistics 41, 173-188.

[11] Gupta, R. D. and Kundu, D. (2001). Exponentiated exponential family: an alternative to gamma and Weibull. Biometrical Journal 43, 117-130.

[12] Gompertz, B. (1825). "On the Nature of the Function Expressive of the Law of Human Mortality, and on a New Mode of Determining the Value of Life Contingencies. Philosophical Transactions of the Royal Society 115: 513585. doi:10.1098/rstl.1825.0026.

[13] Jodra, P. (2009). "A closed form expression for the quantile functions of the Gompertz Makeham distribution". Mathematics and Computers in Simulation 79 (10): 30693075. doi:10.1016/j.matcom.2009.02.002.

[14] Lehmann, E. L. (1953). The power of rank tests. Annals of Mathematical Statistics 24, 23-43. Shahbaz, M. Q., S. Shahbaz and N. S. Butt, 2012. The Kumaraswamy inverse Weibull distribution. Pakistan Journal of Statistics and Operation Research, 8: 479-489.

[15] Makeham, W. M. (1860). "On the Law of Mortality and the Construction of Annuity Tables". J. Inst. Actuaries and Assurance. Mag. 8: 301-310.

[16] Nadarajah, S., and Kotz, S. (2005). The beta exponential distribution. Reliability Engineering and System Safety, 91, 689-697.

[17] Souza, W. M., G. M. Cordeiro and A. B. Simas, 2011. Some results for beta Fréchet distribution. Commun. Statist. TheoryMeth., 40: 798-811.

[18] Zografos, K. and N. Balakrishnan, 2009. On families of betaand generalized gamma-generated distributions and associated inference. Stat. Method., 6: 344-362. s. 